\title{
The role of vaginal-penile sexual intercourses in the skin rejuvenating in postmenopausal women
}

\author{
Piotr Brzezinski ${ }^{1}$, Lorenzo Martin²
}

${ }^{1}$ Institute of Biology and Environmental Protection, Pomeranian Academy, Slupsk, Poland, ${ }^{2}$ University of Siena, Department of Pharmaceutical Biotechnologies, Via A. Moro 2, 53100 Siena, Italy

Corresponding author: Lorenzo Martini, E-mail: martinil163@libero.it

\begin{abstract}
It is well ascertained that sexual intercourse promotes the hypersecretion of estrogens in woman, as well as estrogens are beneficial for skin rejuvenating. This paper, almost revolutionary, focuses its attention on the measurements of TEWL, hydration and electric impedance effectuated on postmenopausal women after three coitus. TEWL is measured by the aids of a tewlmeter, hydration thanks the corneometer and moisture and GAGs gel mosaic of dermal layer owing to the employ of a special apparatus made of 4 electrodes that is apt to evaluate the impedance.
\end{abstract}

Key words: TEWL; Skin moisture; Electric impedance; Sex intercourse; Estrogens

The fact that sexual intercourse encourages the hypersecretion of estrogens in woman is ineluctable

In 2007 some A.A. [1] investigated whether sexual activity was associated with reproductive function and measured the secretion of reproductive hormones in menstruating women, which had regular sexual coitus and heralded that after a vaginal-penile intercourse, serum concentrations of estradiol, luteinizing hormone (LH), follicle-stimulating hormone (FSH), progesterone, and testosterone were measured up to 8 times/cycle, adjusting models for age, race, body mass index, perceived stress, and alcohol consumption.

Elevated concentrations of estrogen $(+14.6 \%)$, luteal progesterone $(+41.0 \%, P<.01)$ and mid-cycle LH $(+23.4 \%, P<.01)$, but not FSH $(P=.33)$ or testosterone $(P=.37)$, were observed by the A.A. in sexually active women compared with sexually inactive women.

Both testosterone and estrogen levels experience a boost through regular sexual activity. Testosterone does more than just boost sex drive, it helps fortify bones and muscles, and it keeps heart in good working condition as well. In women, sex increases the levels of estrogen, which protects against heart disease. This hormone also plays a huge role in a woman's body scent. While estrogen makes women sentimental, a rise in their testosterone levels is what makes them desire penetration $[1,2]$.

Men also produce estrogen and as they age, their estrogen levels increase while their testosterone levels drop. This reversal often results in men becoming somewhat calmer as they get on in years [3].

It is in 2001 that interest in the effects of estrogen has begun progressively to grow. The influence of estrogen on several body systems had been well-documented prior; however, one area that was hitherto not been explored was the effects of estrogen on skin. Estrogen appears to aid in the prevention of skin aging in several ways. This reproductive hormone prevents a decrease in skin collagen in postmenopausal women; in effect topical and systemic estrogen therapy can increase the skin collagen content and therefore maintain skin thickness. In addition, estrogen maintains skin moisture by increasing acid mucopolysaccharides and hyaluronic acid in the skin and possibly maintaining stratum corneum barrier function. Sebum levels

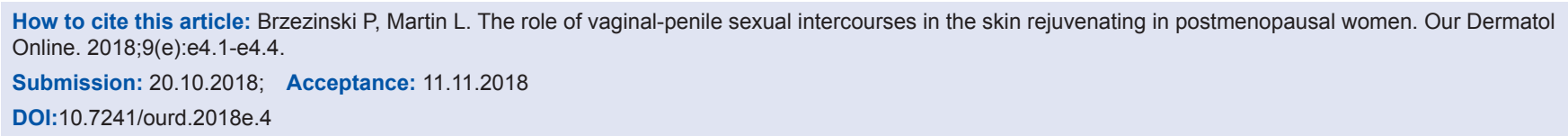


are higher in postmenopausal women receiving hormone replacement therapy. Skin wrinkling also may benefit from estrogen as a result of the effects of the hormone on the elastic fibers and collagen. Outside of its influence on skin aging, it has been suggested that estrogen increases cutaneous wound healing by regulating the levels of a cytokine. In fact, topical estrogen has been found to accelerate and improve wound healing in elderly men and women. The role of estrogen in scarring is unclear but recent studies indicate that the lack of estrogen or the addition of tamoxifen may improve the quality of scarring [4-6].

In this study we have attempted to state how much the hypersecretion of estrogens after a sexual intercourse may interfere with skin beauty and rejuvenating in postmenopausal women, who presented poor skin hydration, low skin moisture and a very high skin electric impedance.

\section{MATERIALS AND METHODS}

We have recruited two gigolos and three postmenopausal women $(55,57$ and 59 y.old, I,II,III in order to let them to have regular sexual intercourses for an entire week end.

It must be considered that each of the two men performed three coitus with each of the three women.

It must be stressed too that I is a white woman, II is a black one and III is Asian.

TEWL, skin hydration and electric impedance was measured at the very beginning of the experiment in all the three women, by the aids of a tewlmeter, a corneometer and an appropriate apparatus build up by 4 electrodes [7].

After each coitus we measured again TEWL, hydration and electric impedance of the three women.

We have employed three instruments to have satisfactory scores concerning the evaluation of three parameters connected with skin conditions:

\section{Evaporimeter (atmometer) to measure TEWL}

Transepidermal water loss (TEWL) is the amount of water that passively evaporates through skin to the external environment due to water vapor pressure gradient on both sides of the skin barrier and is used to characterize skin barrier function. The average TEWL in human is about $300-400 \mathrm{~mL} /$ day; however, it can be affected by environmental and intrinsic factors. In high humidity, the amount of water loss will decrease due to the drop in the water vapor pressure gradient. TEWL varies in different anatomic sites and is inversely related to the corneocyte size. Skin sites with smaller corneocytes have higher TEWL values.

Empirically it is well ascertained that when TEWL increases, skin hydration decreases.

We have used a Tewameter TM 300, that records scores in $\mathrm{g} / \mathrm{h} / \mathrm{m}^{2}$ according to the following equation:

$\mathrm{dm} / \mathrm{dt}=-\mathrm{D} \times \mathrm{A} \times \mathrm{dt} / \mathrm{dx}$

where: $\mathrm{A}=$ surface $\left[\mathrm{m}^{2}\right] ; \mathrm{m}=$ water transported $[\mathrm{g}] ; \mathrm{t}=$ time $[\mathrm{h}] ; \mathrm{D}=$ diffusion constant $[=0.0877 \mathrm{~g} / \mathrm{m}(\mathrm{h}(\mathrm{mmHg}))] ; \mathrm{p}=$ vapour pressure of the atmosphere $[\mathrm{mm} \mathrm{Hg}] ; \mathrm{x}=$ distance from skin surface to point of measurement [m].

This instrument reveals the capacity of measuring the real TEWL at these conditions:

External temperature: $10-40^{\circ} \mathrm{C}$; Relative humidity: $30-70 \%$.

Corneometer to measure the hydration of the stratum corneum: The measurement is based on capacitance measurement of a dielectric medium. We have employed the Corneometer ${ }^{\circledR}$ CM 825 that measures the change in the dielectric constant due to skin surface hydration changing the capacitance of a precision capacitor. The measurement can detect even slight changes in the hydration level.

4 electrodes method to measure the electric impedance [7].

Skin impedance is not but the measure of the degree to which an electric circuit resists electric-current flow when a voltage is impressed across its terminals. Impedance, expressed in Ohms, is the ratio of the voltage impressed across a pair of terminals to the current flow between those terminals. In direct-current (DC) circuits, impedance corresponds to resistance. In alternating current $(\mathrm{AC})$ circuits, impedance is a function of resistance, inductance, and capacitance. Inductors and capacitors build up voltages that oppose the flow of current. This opposition, called reactance, must be combined with resistance to find 
the impedance. The reactance produced by inductance is proportional to the frequency of the alternating current, whereas the reactance produced by capacitance is inversely proportional to the frequency. (we set up the frequency always at $4000 \mathrm{~Hz}$ ).

Many investigators have attempted to utilize the electrical properties of the skin as an indication of the condition of the skin or its underlying tissues. Earliest attempts utilizing direct current encountered the insurmountable problem of polarization currents set up within the body which mask the true electrical resistance of the tissues. Gildemeister [1] was among the first to overcome this difficulty by using an alternating current and measuring the total opposition to its passage through tissue. This total electrical opposition is called impedance and in the skin it has two components, resistance and capacitive reactance.

Where skin is abraded or senescent impedance diminishes and it represents a good marker to determine the good condition of skin.

\section{RESULTS}

In table 1 the scores of TEWL, hydration and electric impedance of the three volunteers (the postmenopausal women) are plotted, after the three sexual intercourses that took place during the week end of delight.

The first measurements of TEWL of the three volunteers at the beginning of the experimentations were: $7.9 ; 6.8 ; 6.1$.

The first measurements of Hydration of the free volunteers at the beginning of the experimentations were: $44,37,31$.

The first measurements of the skin electric impedance of the free volunteers at the beginning of the experimentations were: 1910, 1780, 1590.

\section{CONCLUSIONS}

It is evident that after reiterated sexual coitus in all three the volunteers TEWL diminishes, Hydration electric impedance do increase.

A plentitude of researchers have demonstrated during the last decades [8,9] that Asian epidermis is less prone to senescence and is always more hydrated and

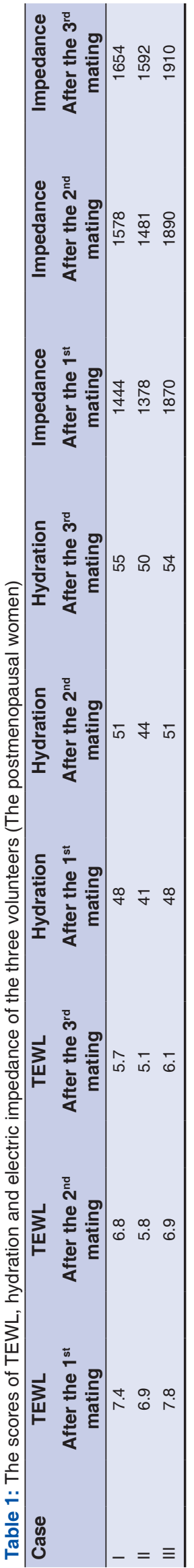


moisturized than white and black epidermis, and in this study this phenomenon is emphasized.

\section{Statement of Human and Animal Rights}

All procedures followed were in accordance with the ethical standards of the responsible committee on human experimentation (institutional and national) and with the Helsinki Declaration of 1975, as revised in 2008.

\section{Statement of Informed Consent}

Informed consent was obtained from all patients for being included in the study.

\section{REFERENCES}

1. Prasad A, Mumford SL, Buck Louis GM, Ahrens KA, Sjaarda LA, Schliep KC, et al. Sexual activity, endogenous reproductive hormones and ovulation in premenopausal women. Horm Behav. 2014;66:330-8

2. aRuiz-Tagle SA, Figueira MM, Vial V, Espinoza-Benavides L,
Miteva M. Micronutrients in hair loss. Our Dermatol Online. 2018;9:320-8

3. 6 Unexpected Health Benefits of Sex:2009; Story.Askmen.com

4. Shah MG, Maibach HI. Estrogen and skin. An overview. Am J Clin Dermatol. 2001;2:143-50.

5. Akdogan N, Dogan S, Atakan N, Yalçin B. Association of serum hormone levels with acne vulgaris: Low estradiol level can be a pathogenetic factor in female acne. Our Dermatol Online. 2018;9:249-56.

6. Chauhan S, Sharma V, Mahajan VK. Urethral caruncle in a young pregnant woman: an uncommon cause of urethral overgrowth. Our Dermatol Online. 2018;9:468-9.

7. Woo EJ, Hua P, Webster JG, Tompkins WJ, Pallás-Areny R. Skin impedance measurements using simple and compound electrodes. Med Biol Eng Comput. 1992;30:97-102.

8. Nouveau S, Yang Z,Mac-Mary S, Lacharrière O. Skin ageing: A comparison between Chinese and European populations - A pilot study. J Dermatol Scien. 2006;40:187-93.

9. La Ruche G, Cesarini JP. Histology and physiology of black skin; Ann Dermatol Venereol. 1992;119:567-74.

Copyright by Piotr Brzezinski, et al. This is an open-access article distributed under the terms of the Creative Commons Attribution License, which permits unrestricted use, distribution, and reproduction in any medium, provided the original author and source are credited.

Source of Support: Nil, Conflict of Interest: None declared. 